\title{
Comparative study of three different BACTEC culture media for the detection of bacteremia in ambulatory and hospitalized children
}

Deirdre L Church MD, H Dele Davies MD, G Cadrain RT, Cynthia L Trevenen MD

\begin{abstract}
DL Church, HD Davies, G Cadrain, CL Trevenen. Comparative study of three different BACTEC culture media for the detection of bacteremia in ambulatory and hospitalized children. Can J Infect Dis 1998;9(2):77-82.

To compare the yield of two aerobic and an anaerobic BACTEC blood culture media in detecting bacteremia in ambulatory and hospitalized care settings at a children's hospital, a prospective cohort study was completed. Over an 18-month period, equal blood volumes (minimum of $1 \mathrm{~mL} /$ bottle) were inoculated into a three-bottle culture set including aerobic BACTEC NR 6A, aerobic BACTEC PEDS Plus and anaerobic NR 7A broths. Chart reviews were completed on all children with bacteremia to determine whether the isolate was clinically significant based on predefined criteria. Among 5328 evaluable blood culture sets, 323 clinically significant organisms ( 110 from ambulatory and 213 from hospitalized children) were isolated. Most Streptococcus pneumoniae, Haemophilus species, and Neisseria or Moraxella species were recovered from children attending the emergency department or out-patient clinics. Important isolates in hospitalized children included most of the staphylococci and Enterobacteriaceae, and all group D enterococci, Gram-negative nonfermentative bacilli and all Candida species. Overall, significantly more isolates were detected only in the anaerobic bottle from ambulatory children $(\mathrm{P}<0.0001)$, including 13 of $54(24 \%)$ patients with $S$ pneumoniae bacteremias presenting to the emergency department. This study indicated that different BACTEC blood culture media combinations are needed in ambulatory and hospitalized pediatric care settings to ensure the optimal recovery of all types of isolates. Whereas aerobic blood culture bottles are adequate for detection of bacteremia in hospitalized children, the common occurrence of fastidious organisms mandates the need for a combined aerobic/anaerobic culture set in ambulatory pediatric care settings.
\end{abstract}

Key Words: Ambulatory care, Bacteremia, Children, Hospitalization

Étude comparative de trois milieux de culture BACTEC différents pour le dépistage de la bactériémie chez des enfants hospitalisés ou non

RÉSUMÉ : On a effectué une étude de cohorte prospective afin de comparer le rendement de deux milieux d'hémocultures BACTEC aérobies et un anaérobie pour le dépistage de la bactériémie dans des hôpitaux pédiatriques chez les enfants hospitalisés ou non. Sur une période de 18 mois, des volumes égaux de sang (minimum de $1 \mathrm{~mL} / \mathrm{flacon}$ ) ont été voir page suivante

Presented in part at the 94th General Meeting of the American Society for Microbiology, May 1994, Washington DC Departments of Microbiology and Infectious Diseases, Pediatrics, Pathology, University of Calgary; and Department of Clinical Laboratories (Microbiology) and The Child Health Research Unit, Alberta Children's Hospital, Calgary, Alberta

Correspondence and reprints: Dr DL Church, Calgary Laboratory Services, 1638-10th Avenue SW, Calgary, Alberta T3C OJ3. Telephone 403-209-5281, fax 403-209-5347, e-mail dchurch@cml.ab.ca

Received for publication February 3, 1997. Accepted April 24, 1997 
injectés dans un nécessaire pour hémocultures comportant trois flacons, soit les bouillons pour cultures aérobies BACTEC NR 6A (6A), BACTEC PEDS Plus (PP) et anaérobie NR 7A (7A). L'étude des dossiers a été complétée pour tous les enfants atteints de bactériémie afin de déterminer si l'isolat était cliniquement significatif en fonction des critères prédéfinis. Parmi les 5328 nécessaires pour hémocultures évaluables, 323 organismes cliniquement significatifs ont pu être isolés (110 de patients ambulatoires et 213 de patients hospitalisés). La plupart des espèces de Streptococcus pneumonia, d'Homophilus et de Neisseria ou Moraxella ont été isolées chez des enfants vus au service des urgences ou dans les cliniques ambulatoires. Les isolats prélevés chez les enfants hospitalisés ont été la plupart des staphylocoques et des enterobacteriacea et tous les entérocoques du groupe $\mathrm{D}$, des bacilles gram-négatifs ou non fermentescibles et toutes les espèces de Candida. De façon globale, un nombre significativement plus grand d'isolats ont été décelés seulement dans les flacons anaérobies prélevés chez des enfants non hospitalisés $(P 0,001)$, y compris 13 patients sur $54(24 \%)$ souffrant de bactériémie à $S$. pneumonice vus au service des urgences. Cette étude a indiqué que les différentes combinaisons de milieux d'hémocultures BACTEC sont nécessaires pour les soins pédiatriques chez les enfants hospitalisés ou non afin d'assurer l'identification optimale de tous les types d'isolats. Les flacons pour hémocultures aérobies conviennent au dépistage de la bactériémie chez les enfants hospitalisés, mais la fréquence des organismes fastidieux exige le recours à une trousse pour hémoculture combinée aérobie/anaréobie pour la population pédiatrique non hospitalisée.

$\mathrm{B}_{\mathrm{b}}^{\mathrm{a}}$ acteremic illness in children must be rapidly diagnosed by culturing blood. Optimal detection of pediatric bacteremia by a laboratory must address the need to recover a much broader range of pathogens than are generally found in adults. In addition to all of the major pathogens normally recovered from hospitalized patient populations, ambulatory children are also more often infected with fastidious pathogens such as Streptococcus pneumonias, Haemophilus influenzas and Neisseria meningitidis (1). The total blood volume that safely and feasibly can be obtained from young children in a single phlebotomy effort is also much less than that which can be taken from an adult, ie, in most cases, 1 to $1.5 \mathrm{~mL}$ per bottle is inoculated into a pediatric blood culture versus up to $10 \mathrm{~mL}$ per bottle for adults.

To enhance the detection of bacteremia, it has been routine practice for clinical microbiology laboratories servicing children to draw a paired blood culture set including an aerobic plus an anaerobic media (2). This policy has recently been challenged by several pediatric studies that have recommended the use of only paired aerobic media with the addition of an anaerobic medium in certain clinical situations (3-5). Weisse et al (6) on the other hand recently recommended that a three-bottle draw (paired aerobic plus an anaerobic media) be done for children. However, none of these studies distinguished between the recovery of bacteremic isolates from ambulatory children in whom fastidious pathogens are frequently recovered versus those who had been hospitalized.

Although children have a low incidence of significant anaerobic infection (2-7), the role of the anaerobic media for the enhanced isolation of fastidious organisms needed to be clarified. Previous experience at our hospital suggested that many clinically significant $S$ pneumoniae cases were only being recovered in the anaerobic blood culture bottle. A prospective cohort blood culture study was performed to compare the yield of different bacteremia pathogens from ambulatory and hospitalized children from two aerobic and one anaerobic blood culture media.

\section{PATIENTS AND METHODS}

Patient population: The Alberta Children's Hospital is a tertiary regional referral centre in Calgary, Alberta, which is affiliated with the Faculty of Medicine, University of Calgary. It is the western Canadian site for pediatric bone marrow transplantation. The hospital serves a population base of 1.2 million people, seeing children referred from southern Alberta, southeastern British Columbia and southwestern Saskatchewan. In an average year, approximately 6700 children are hospitalized, 36,000 children are seen through the emergency department and another 60,000 children visit various subspecialty clinics and physicians' offices. All children having blood drawn for culture between March 1, 1992 and August 31, 1993 were enrolled in this study. The study was approved by the Conjoint Medical Ethics Committee of the University of Calgary.

Blood samples: Blood was drawn from pediatric patients with suspected bacteremia by a trained phlebotomy team. A total of 3 to $5 \mathrm{~mL}$ of blood were drawn per child, and then 1 to $1.5 \mathrm{~mL}$ aliquots were inoculated into each of three blood culture media types. This set comprising conventional BACTEC NR 6A (6A), conventional BACTEC NR 7A (7A) and BACTEC PEDS Plus (PP) (all Becton-Dickinson). Fluid levels in each of the inoculated bottles were assessed to ensure that equivalent volumes of blood had been inoculated into each bottle of the culture set. If fluid levels could not be accurately assessed, inoculated bottles were reweighed and the preinoculated weight was subtracted from the total inoculated bottle weight.

Blood culture media: $6 \mathrm{~A}$ is a $30 \mathrm{~mL}$ nonresinated aerobic soybean-casein digest broth base containing $0.035 \%$ sodium polyanethole sulphonate (SPS). 7A is also a $30 \mathrm{~mL}$ nonresinated media with a similar SPS concentration, but the medium is prereduced and the atmosphere in the bottle is anaerobic. PP broth is composed of a conventional aerobic $6 \mathrm{~A}$ soybeancasein digest broth base, modified by inclusion of nonionic absorbing resins, cationic exchange resins and $0.06 \%$ yeast extract. In addition, the concentration of SPS is decreased from 0.035 to $0.025 \%$, and the broth volume is $10 \mathrm{~mL}$.

Laboratory blood culture protocol: The BACTEC NR 730 automated blood culture system (Becton-Dickinson, Maryland) was used for this study. All blood culture bottles were incubated at 35 to $37^{\circ} \mathrm{C}$ for up to seven days, with shaking during the first $48 \mathrm{~h}$. During this time, the vials were examined visually and tested twice (once in the morning and again in the afternoon). Thereafter, they were examined and tested on a daily basis. Any bottle showing visible growth (ie, hemolysis, turbidity or excess gas production causing the vial septum to bulge) was 
TABLE 1

Recovery of clinically significant isolates in aerobic and/or anaerobic medium from ambulatory children

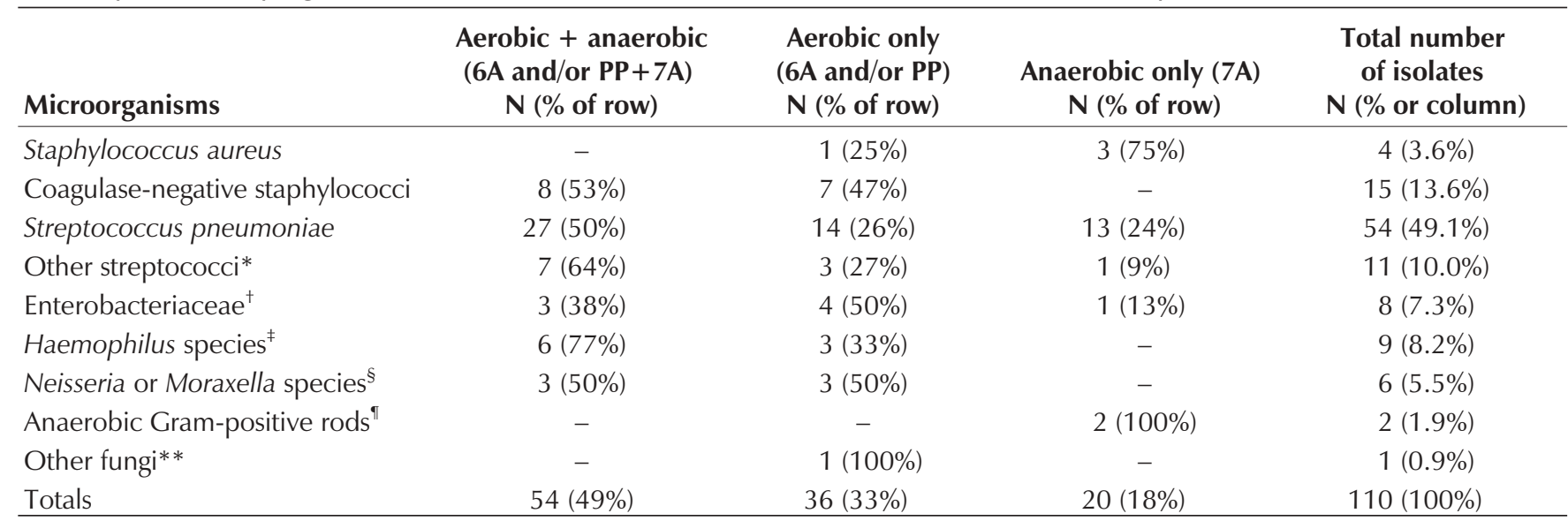

*Includes four viridans streptococci, three Streptococcus pyogenes (group A streptococci), two Streptococcus agalactiae (group B streptococci), one group C streptococcus, one group G streptococcus; ${ }^{+}$Includes three Salmonella typhi, four Escherichia coli, one other Salmonella species; ${ }^{\ddagger}$ Includes eight Haemophilus influenzae type $b$, one Haemophilius parainfluenzae; ${ }^{\S}$ Includes five Neisseria meningitidis, one other Neisseria species; ${ }^{\pi}$ Includes one Actinomyces viscosus, one Propionibacterium acnes; **One Malassezia furfur. 6A BACTEC NR 6A aerobic medium; 7A BACTEC NR 7A anaerobic medium; PP BACTEC PEDS Plus

not read on the instrument, but investigated immediately as a positive culture. In accordance with the manufacturer's instructions, all bottles showing positive growth values on the BACTEC were immediately removed for further testing. Growth values set for the instrument for aerobic (6A or PP bottles) and anaerobic (7A) blood culture bottles were 20 units or more. A positive culture was also indicated by an increase in the growth value of 10 or more between consecutive readings for all bottle types. If one or more of the bottles $(6 \mathrm{~A}+7 \mathrm{~A}, 6 \mathrm{~A}+\mathrm{PP}$, $\mathrm{PP}+7 \mathrm{~A})$ in a set became positive either visually or by an increased growth value, all of the other bottles in the set were also tested (Gram stain and/or acridine orange stain plus subculturing to plates). After testing, any detected and confirmed positive vials were removed from the system, and all other bottles were returned to the incubator for further reading. The first bottle(s) to become positive was recorded. Only blood cultures from children suspected by their attending physician of having osteomyelitis, endocarditis or fungemia were read daily for an extended time period (ie, minimum of 14 days) before being reported as negative. Terminal Gram and/or acridine orange stains were done on all blood cultures.

Compliant sets: Compliant blood culture sets that qualified for evaluation included sets where all of the three bottles were inoculated, sets where equivalent blood volumes had been inoculated in each bottle as outlined above (see Patients and Methods - Blood samples), and sets that were handled strictly according to the laboratory protocol.

Definition of clinically significant isolates: Chart reviews on all children with positive blood cultures were completed by one investigator to determine whether the isolate was clinically significant. Ambulatory children were defined as out-patients and those attending the emergency department. Hospitalized children were defined as those who had been admitted to the hospital for at least $24 \mathrm{~h}$. Clinically significant isolates fulfilled at least one of the following criteria: clinical signs and symptoms of infection (ie, one of fever, elevated peripheral white blood cell count or left shift), or a confirmed site or source of infection; a significant pathogen isolated from any of the three blood culture bottles; the same organism isolated from two or more bottles in a given blood culture set; or the same organism isolated in one or more bottles from more than one blood culture set. The most common blood culture contaminants included coagulase negative staphylococci, Streptococcus viridans and Bacillus species (see Results), with all other isolates being deemed significant pathogens. All antibiotics prescribed at the time that the blood cultures were drawn were also recorded.

Statistical analysis: Blood culture data were entered into a blood culture database program (Becton-Dickinson) to obtain descriptive data (ie, summary report, recovery report, etc). To avoid bias due to multiple comparisons, a statistical test was performed on only the overall difference between isolates recovered in the ambulatory and hospitalized settings. The $95 \%$ confidence interval for the difference in group proportions (delta) was calculated using the binomial distribution. Statistical significance was $\mathrm{P}<0.05$. The normal approximation to the binomial distribution was used for calculating the $95 \%$ confidence interval for single proportions.

\section{RESULTS}

Evaluable sets of blood cultures: Among 6118 sets of blood cultures submitted to the laboratory, 5328 (87\%) sets were evaluated. Thirteen per cent of the total number of sets (790 of 6118) enrolled were excluded because they did not meet the compliant set criteria. The overall rate of recovery (49 of 790 , $6.2 \%$ ) of clinically significant bacteremia isolates in the discarded blood culture sets was similar to that of isolates included in the study. Of the compliant sets, 1864 of 5328 (35\%) were drawn from ambulatory children compared with 3464 of 5328 (65\%) from hospitalized children. A total of 407 of 5328 ( $8 \%$ ) of the sets were culture positive. However, after a review of the individual children's records, $84(1.6 \%)$ isolates were not 
TABLE 2

Recovery of clinically significant isolates in aerobic and/or anaerobic medium from hospitalized children

\begin{tabular}{|c|c|c|c|c|}
\hline Microorganisms & $\begin{array}{c}\text { Aerobic }+ \text { anaerobic } \\
(6 \mathrm{~A} \text { and/or } \mathrm{PP}+7 \mathrm{~A}) \\
\mathrm{N}(\% \text { of row })\end{array}$ & $\begin{array}{c}\text { Aerobic only } \\
\text { (6A and/or PP) } \\
\text { N (\% of row) }\end{array}$ & $\begin{array}{c}\text { Anaerobic only (7A) } \\
\text { N (\% of row) }\end{array}$ & $\begin{array}{c}\text { Total number } \\
\text { of isolates } \\
\mathrm{N} \text { (\% of column) }\end{array}$ \\
\hline Staphylococcus aureus & $12(50 \%)$ & $9(38 \%)$ & $3(12 \%)$ & $24(11.3 \%)$ \\
\hline Coagulase-negative staphylococci & $54(87 \%)$ & $8(13 \%)$ & - & $62(29.1 \%)$ \\
\hline Streptococcus pneumoniae & $4(100 \%)$ & - & - & $4(1.9 \%)$ \\
\hline Other streptococci* & $17(81 \%)$ & $3(14 \%)$ & $1(5 \%)$ & $21(9.9 \%)$ \\
\hline Pediococci & $1(100 \%)$ & - & - & $1(0.5 \%)$ \\
\hline Group D enterococci & $12(80 \%)$ & $2(13 \%)$ & $1(7 \%)$ & $15(7.0 \%)$ \\
\hline Aerobic Gram-positive rods $^{\dagger}$ & $3(60 \%)$ & $1(20 \%)$ & $1(20 \%)$ & $5(2.3 \%)$ \\
\hline Enterobacteriaceae $^{\ddagger}$ & $26(87 \%)$ & $3(10 \%)$ & $1(3 \%)$ & $30(14.1 \%)$ \\
\hline $\begin{array}{l}\text { Gram-negative rods } \\
\text { (nonfermentors) }\end{array}$ & $10(63 \%)$ & $6(37 \%)$ & - & $16(7.5 \%)$ \\
\hline Haemophilus species" & $1(50 \%)$ & $1(50 \%)$ & - & $2(0.9 \%)$ \\
\hline Neisseria or Moraxella species** & $1(50 \%)$ & $1(50 \%)$ & - & $2(0.9 \%)$ \\
\hline Candida species ${ }^{\dagger+}$ & $25(86 \%)$ & $4(14 \%)$ & - & $29(13.6 \%)$ \\
\hline Other fungi $^{\ddagger \neq}$ & - & $2(100 \%)$ & - & $2(0.9 \%)$ \\
\hline Totals & $166(78 \%)$ & $40(19 \%)$ & $7(3 \%)$ & $213(100 \%)$ \\
\hline
\end{tabular}

*Includes 15 Streptococcus viridans, four Streptococcus pyogenes (group A streptococci), two Streptococcus agalactiae (group B streptococci); ${ }^{\dagger}$ Includes five Corynebacterium species; ${ }^{\ddagger}$ Includes eight Escherichia coli, eight Enterobacter species, three Citrobacter freundii, seven Klebsiella pneumoniae, four Proteus mirabilis; ${ }^{\S}$ Includes five Pseudomonas aeruginosa, four other Pseudomonas species, six Acinetobacter species, one Flavobacterium species; ${ }^{\circ}$ One Haemophilus parainfluenzae; ${ }^{* *}$ Includes one Neisseria species, one Moraxella catarrhalis; ${ }^{{ }^{+}}$Includes 25 Candida albicans, four Torulopsis glabrata; ${ }^{\text {\#* } I n-}$ cludes one Aspergillus fumigatus, one Penicillium species. 6A BACTEC NR 6A aerobic medium; 7A BACTEC NR 7A anaerobic medium; PP BACTEC PEDS Plus

deemed to be clinically significant (55 of 84 [65\%] coagulase negative staphylococci, 19 of 84 [23\%] $S$ viridans, six of 84 [7\%] Bacillus species, and four of 84 [5\%] of each one of Micrococcus species, Aerococcus species, Propionibacterium acnes and Neisseria sicca). This left a total of 323 evaluable, clinically significant isolates, which had been recovered from 258 children (ie, 65 children had two or more positive blood culture sets for a single episode of bacteremia).

Recovery of clinically significant bacteremia isolates from ambulatory and hospitalized children: Overall, 220 of 323 $(68 \%)$ clinically significant isolates were recovered simultaneously in one or both aerobic (6A and/or PP) and the anaerobic (7A) media, while another 76 of 323 (24\%) were only recovered in one or both of the aerobic media from both ambulatory and hospitalized children. Overall, a paired aerobic bottle set $(6 \mathrm{~A}$ and PP) detected 298 of 323 (92\%) of all isolates, with the remaining 25 of 323 (8\%) isolates only being recovered in the anaerobic media.

Table 1 outlines the recovery of different types of clinically significant bacteremia isolates from ambulatory children. Approximately one-third of all blood culture isolates were recovered from ambulatory children, and the overall positive rate in this setting was 110 sets of the 1864 (6\%) drawn. Most fastidious pathogens including almost all of the Staphylococcus pneumoniae, Haemophilus species and Neisseria species isolates were recovered from ambulatory children. Neisseria meningitisis was recovered only from children presenting to the emergency department with a febrile illness or meningitis, and meningococci were the most commonly identified Neisse- ria species from ambulatory children. Salmonella species infections accounted for one half of the Enterobacteriaceae isolated. Infections caused by other streptococci included three children with invasive group A streptococcal infections as well as two infants with bacteremia due to group B streptococci. The only anaerobic isolates were a Propionibacterium acnes bacteremia in a child with a cerebrospinal fluid shunt site infection who was admitted through the emergency department, and an Actinomyces viscosus isolated from a child with intra-abdominal infection.

Almost one-half of the clinically significant isolates recovered from ambulatory children were simultaneously found in an aerobic (6A and/or PP) medium and the anaerobic (7A) media, while another one-third were only recovered in one or both of the aerobic bottles (Table 1). However, utilization of only aerobic blood culture media in the ambulatory setting would have missed $18 \%$ (20 of 110 ) of the total number of isolates. Overall, significantly more isolates were detected only in the anaerobic bottle from ambulatory children (20 of $110,18.2 \%, 95 \mathrm{CI}=11.0 \%$ to $25.4 \%$ ) versus hospitalized children (seven of $213,3.3 \%, 95 \% \mathrm{CI}=0.9 \%$ to $5.7 \%$ ). This difference between isolation rates in anaerobic bottles alone from ambulatory compared with hospitalized patients (delta = $18.2 \%-3.3 \%=14.9 \%)$ was highly significant $(\mathrm{P}<0.0001,95 \% \mathrm{CI}$ of delta $=5.8 \%$ to $20.4 \%$ ). This translates into a relative risk of 5.5 (18.2 divided by 3.3 ) of isolation of an organism from the anaerobic medium alone in the ambulatory setting compared with the in-patient setting. A large proportion of $S$ pneumoniae bacteremias in particular ( 13 of $54,124 \%, 95 \% \mathrm{CI}=12.7 \%$ 
to $35.5 \%$ ) were detected only in the anaerobic medium among out-patients. In addition, bacteremia would not have been detected in one child with invasive group A streptococcal infection and another child with typhoid fever. Fungi were infrequently isolated from out-patients, with only one Malassezia furfur infection detected.

Although almost twice as many isolates were recovered from hospitalized children, the overall rate of positive blood culture sets of 213 of 3464 (6.2\%) was similar to that found for ambulatory children (Table 2). However, many more different types of bacterial isolates were recovered, including most coagulase negative staphylococci, $S$ aureus and Enterobacteriaceae, as well as all group D enterococci and Gram-negative bacilli (nonfermentative) and Candida species. Pseudomonas aeruginosa and other Gram-negative nonfermentative bacilli as well as Candida species were mainly found in immunocompromised children (eg, on the oncology ward). Fastidious pathogens (ie, S pneumoniae, Haemophilus species, Neisseria species) were only recovered when a critically ill child was directly admitted to the intensive care unit before blood cultures were drawn. Fungemia aside from Candida species isolates were only found in two children admitted to the oncology ward.

Effect of antibiotic therapy: A total of 105 of the isolates were recovered from children who were on either oral or parenteral antibiotic therapy (data not shown). Because all of these children had a single positive blood culture, almost one-half (105 of $258,41 \%$ ) of all children with positive culture were on antibiotics when their blood cultures were drawn. Only 40 of 105 $(38 \%)$ isolates were recovered in both aerobic and the anaerobic media, with another 29 of 105 (28\%) detected in two media types (ie, $6 \mathrm{~A}+7 \mathrm{~A}, 6 \mathrm{~A}+\mathrm{PP}$ or $\mathrm{PP}+7 \mathrm{~A}$ ). Overall, paired aerobic media again recovered most of the isolates ( 92 of $105,88 \%$ ). As a single medium, PP enhanced the recovery of coagulase negative staphylococci and $S$ aureus as well $N$ meningitidis in children already being given antibiotics.

\section{DISCUSSION}

Bacteremia is one of the most serious infectious diseases of childhood and may be caused by a wide variety of microorganisms. Although it is well established that different types of bacteremia pathogens are recovered from ambulatory and hospitalized children (1), our large prospective study demonstrates that the use of different types of BACTEC blood culture media is important to optimize the recovery of different etiological agents. Some recently reported pediatric blood culture studies have recommended discontinuing the routine use of anaerobic media, but none of these studies differentiated recovery rates of different pathogens between the ambulatory and hospitalized pediatric care settings (3-5). Although our study confirms that children have a very low rate of anaerobic bacteremia (2-7), use of the BACTEC anaerobic media was essential for optimal recovery of $S$ pneumoniae as well as other streptococci and Salmonella species in ambulatory children. Because streptococci and Enterobacteriaceae are facultative organisms, the reduced environment of the BACTEC anaerobic media likely enhances the recovery of organisms in their early growth phase at the time of bottle inoculation. Whether enhanced recovery of these types of bacteria is found in anaerobic media from other blood culture systems (eg, BacT/Alert, Organon-Teknika, North Carolina) must be independently studied.

The total blood volume drawn from a child is also important to the overall recovery rate of isolates from blood cultures as has previously been established in adults (8-10). Although our study was not designed to evaluate the role of blood volume, a recent three-bottle pediatric study by Isaacman et al (11) indicates that culturing an increased amount of blood enhances the early recovery of organisms. This study also demonstrated that use of the anaerobic media increased the yield of pneumococci by as much as $12 \%$. A study by Weisse et al (6) also evaluated a three-bottle blood culture set in a pediatric cohort and found that there was an incremental increase in the recovery of bacteria with the use of single versus multiple bottle sets. Thus, it appears from our study and others that culturing an increased blood volume also affects the overall recovery of not only fastidious organisms such as $S$ pneumoniae, but also a wide range of other isolates. Although increased amounts of blood should be taken from children for blood cultures, the optimal amounts to be taken from infants and children of various ages remains to be determined.

Based on our study and those of others, we recommend that a paired aerobic plus an anaerobic media blood culture set be routinely drawn from ambulatory children to optimize the recovery of $S$ pneumoniae and other facultative and/or fastidious pathogens. Because of the frequent use of antibiotics in febrile young children presenting to ambulatory settings (2) (almost half in our study), PP or other media with nonionic absorbing resins should be the aerobic medium of choice. For hospitalized children, our data support the use of a paired aerobic media set without routinely using an anaerobic medium. However, to make the diagnosis of pediatric bacteremia as cost effective as possible, a study comparing the recovery of different bacteremia pathogens in hospitalized children after inoculation of a standardized amount of blood into a paired aerobic media set and inoculation of the entire amount of blood into a single 'higher' volume aerobic bottle should be completed. Evaluations also need to be undertaken to establish the optimal amount of blood that should be drawn from children of different ages, including neonates, to optimize the recovery of pediatric bacteremia isolates.

\section{CONCLUSIONS}

In hospitalized children, a paired aerobic BACTEC blood culture media set (eg, PP and 6A) yields most of the significant pathogens and is adequate in the majority of situations. A single large volume aerobic bottle may also be equivalent but needs to be evaluated (11). The role of the anaerobic media (7A) should be reserved for clinical situations suggesting a higher likelihood of bacteremic anaerobic infections according to the guidelines reported by Zaidi et al (5). For ambulatory children, an anaerobic blood culture medium should be included in the blood culture set to optimize the recovery of fastidious pathogens such as $S$ pneumoniae. 
ACKNOWLEDGEMENTS: We thank Valerie Sim for assistance with the data entry. Dr HD Davies is a Clinical Investigator of the Alberta Heritage Foundation for Medical Research. The study was funded by the Alberta Children's Hospital Clinical Laboratories, and was partly supported by Becton-Dickinson.

\section{REFERENCES}

1. Kaplan SL. Bacteremia and endotoxin shock. In: Feigin RD, Cherry JD, eds. Textbook of Pediatric Infectious Diseases. Philadelphia: WB Saunders Co, 1992:863-75.

2. Morello JA, Matushek SM, Dunne WM, Hinds DB. Performance of a BACTEC nonradiometric media for pediatric blood cultures.

Clin Microbial 1991;29:359-62.

3. Murray PR, Traynor P, Hopson D. Critical assessment of blood culture techniques: analysis of recovery of obligate and facultative anaerobes, strict aerobic bacteria, and fungi in aerobic and anaerobic blood culture bottles. J Clin Microbiol 1992;30:1462-8.
4. Dunne WM, Tillman J, Havens PL. Assessing the need for anaerobic media for recovery of clinically significant blood culture isolates in children. Pediatr Infect Dis J 1994;13:203-6.

5. Zaidi AKM, Knaut AL, Mirrett S, Reller LB. Value of routine anaerobic blood cultures for pediatric patients. J Pediatr 1995;127:263-8.

6. Weisse ME, Bass JW, Young LM. Pediatric blood culture: comparison of yields using aerobic, anaerobic and hypertonic media. Pediatr Infect Dis J 1992;11:123-5.

7. Henry NK, McLimans CA, Wright AJ, Thompson RL, Wilson WR, Washington JA II. Microbiological and clinical evaluation of the isolator lysis-centrifugation blood culture tube. J Clin Microbiol 1983;17:864-9.

8. Werner AS, Cobbs CG, Kaye D, Hook EW. Studies on the bacteraemia of bacterial endocarditis. JAMA 1967;202:199-203.

9. Dietzman DE, Fisher GW, Schoenknecht FD. Neonatal Escherichia coli septicemia - bacterial counts in blood. J Pediatr 1974;85:128-30.

10. Yagupsky P, Nolte FS. Quantitative aspects of septicemia. Clin Microbiol Rev 1980;3:269-79.

11. Isaacman DJ, Karasic RB, Reynolds EA, Kost SI. Effect of number of blood cultures and volume of blood on detection of bacteraemia in children. J Pediatr 1996;128:190-5. 


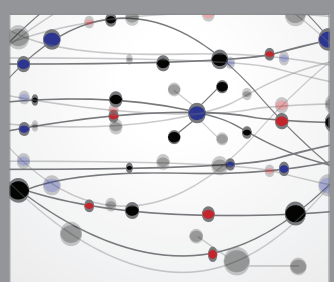

The Scientific World Journal
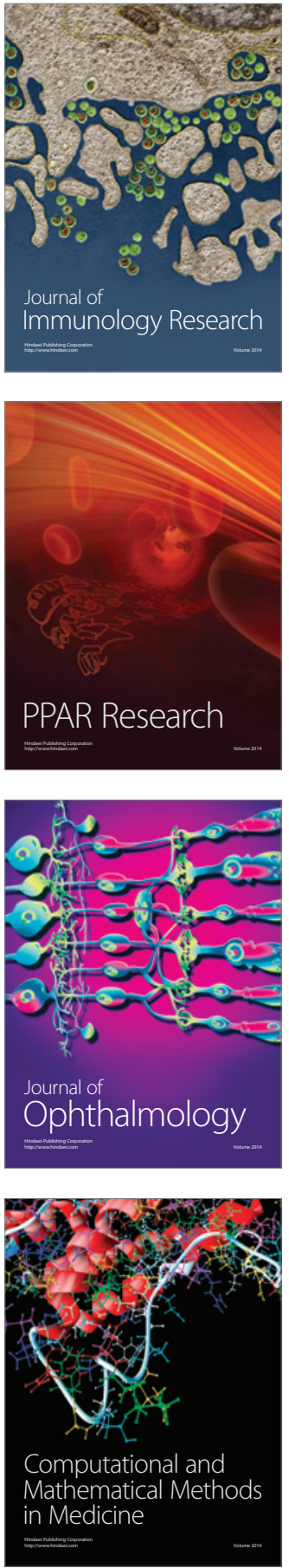

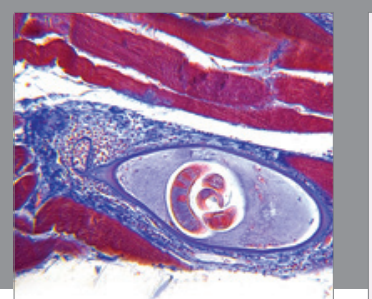

Gastroenterology Research and Practice

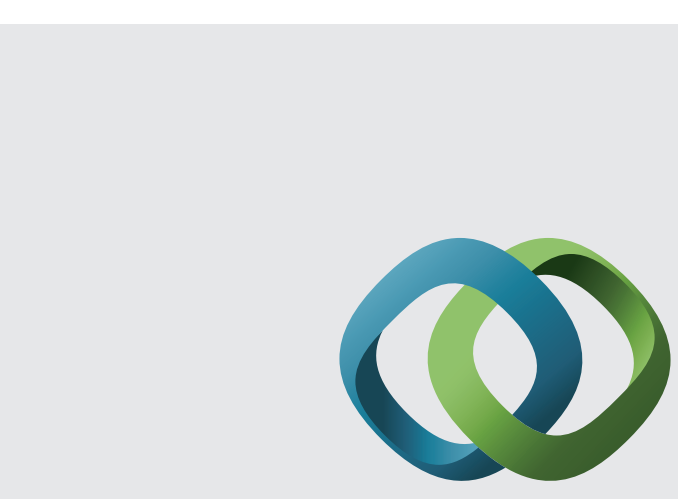

\section{Hindawi}

Submit your manuscripts at

http://www.hindawi.com
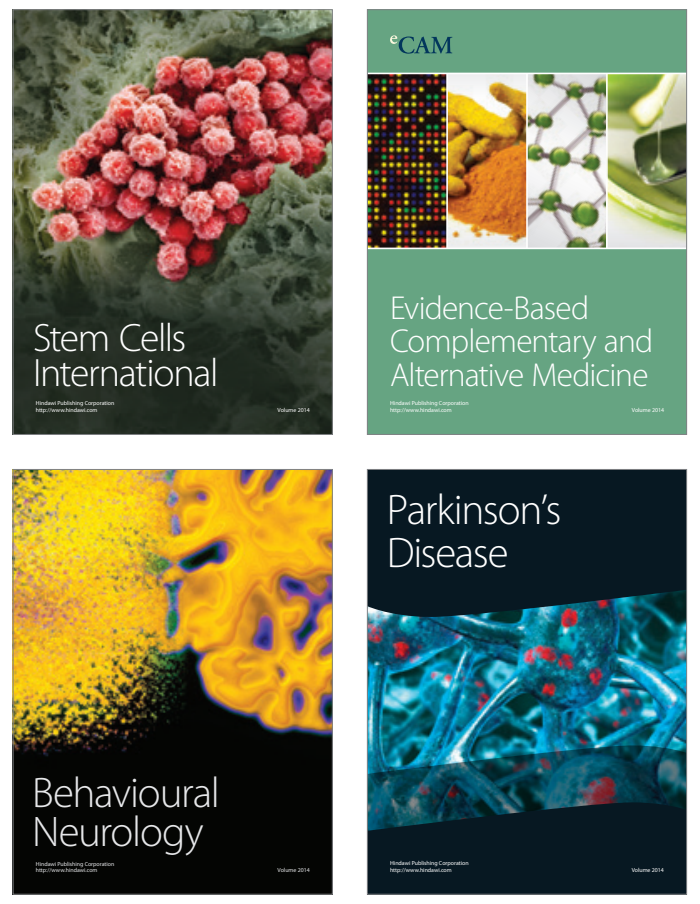
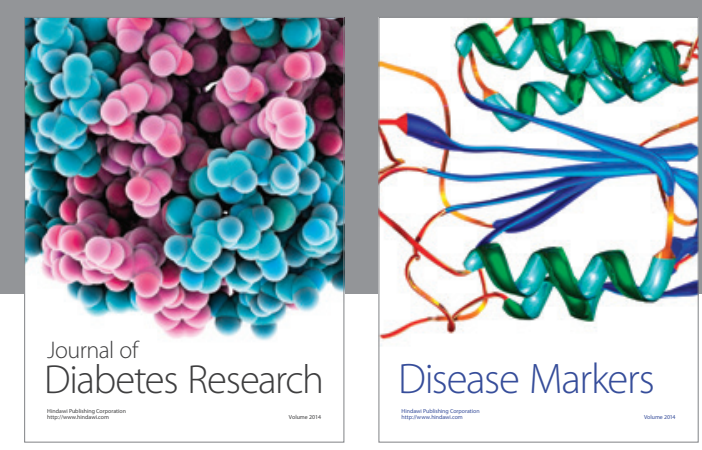

Disease Markers
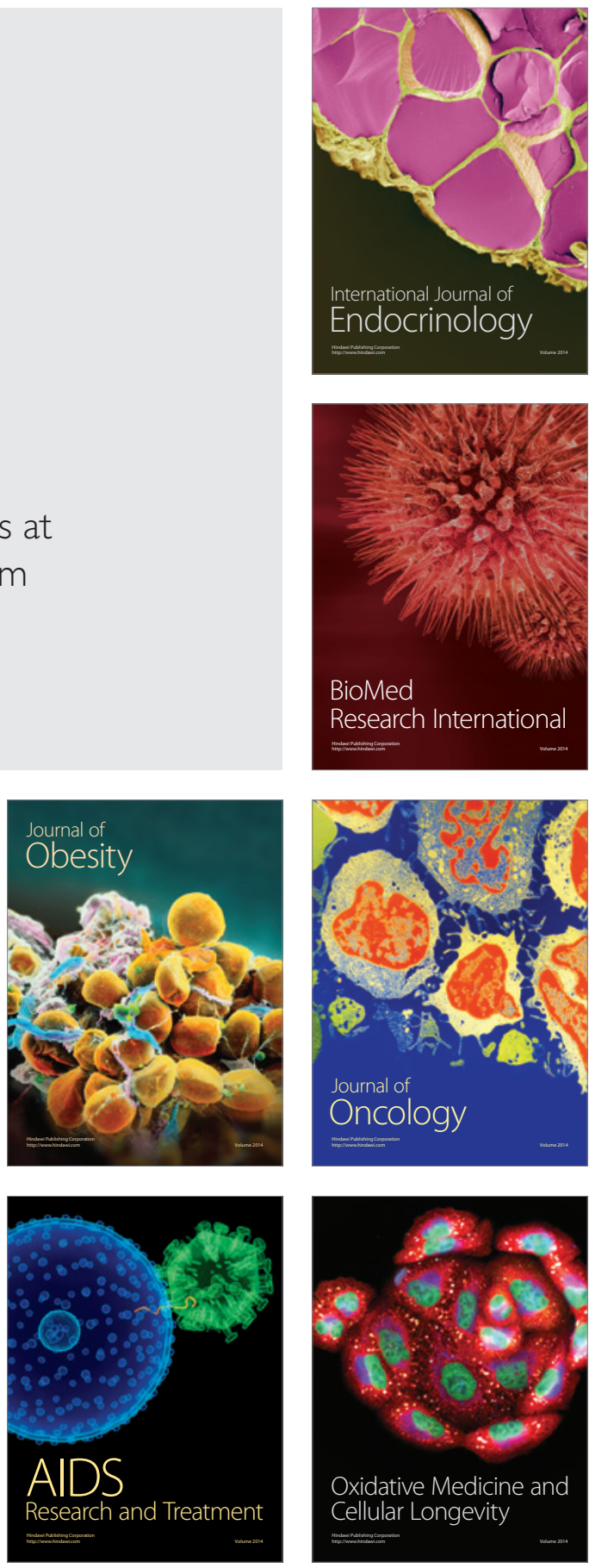